\title{
MORTALIDADE PERINATAL EM SÃO PAULO, BRASIL
}

Ruy Laurenti *

Maria Helena Silveira*

Arnaldo A. F. Siqueira**

\begin{abstract}
RSPU-B/253
Laurenti, R. et al. - Mortalidade perinatal em São Paulo, Brasil. Rev.

Saúde públ., S. Paulo, 9:115-24, 1975.

Resumo: Foi analisada a mortalidade verinatal em são Paulo num periodo de dois anos. Partiu o estudo da totalidade dos atestados de nascidos mortos e de uma amostra de b́bitos de menores de sete dias, para a qual a metodologia foi a de entrevistas domiciliares e junto aos médicos e hospitais que tenham prestado assistência às crianças falecidas. O coeficiente de mortalidade perinatal encontrado foi igual a 42,04 por mil nascidos vivos. Esse valor apresenta-se bastante elevado quando comparado ao de areas desenvolvidas. Foi verificado que ele poderia ser diminuído com a simples redução dos coeficientes especificos por algumas causas evitáveis a nivel de pré-natal (sífilis congênita, doenças próprias ou associadas à gravidez), do parto (distócias, traumatismos obstétricos e anóxia), ou da atenção ao recém-nascido (causas infecciosas, do aparelho respiratório, hemorragias e certas anóxias). o coeficiente de mortalidade perinatal segundo a idade da mãe mostrou que o risco varia com a idade, apresentando-se maior nas mulheres de 40 a 49 anos.
\end{abstract}

UnItermos: Mortalidade perinatal, São Paulo, Brasil. Estatistica vital. Mortalidade neonatal. Natimortalidade.

$O$ coeficiente de mortalidade neonatal tem sido utilizado, desde há muito tempo, como indicador de saúde de uma população. No entanto, como mede a força dos diferentes fatores que influem na mortalidade dos recém-nascidos, reflete também a qualidade da atenção dada a estes, ao parto e a nível de pré-natal. Vale dizer que numa região que propicie à população um bom atendimento desde o pré-natal, ao parto e à criança, fatalmente a mortalidade neonatal será baixa. É o que se verifica nos países desenvolvidos, o contrário podendo ser visto nas regiōes em desenvolvimento, onde se inclui o Brasil.

Mais recentemente, porém, tem-se observado que a maioria das causas de morbidade e mortalidade que afetam o recém-nascido, principalmente na primeira semana de vida, incidem também sobre o feto "in utero", ou seja, as causas de mortalidade neonatal são, em geral, as mesmas causas da natimortalidade. Essa identidade de problemas exigiu a união do fim do período pré-natal ao início do neonatal, caracterizando o período peri-

* Da Disciplina de Estatistica Vital do Departamento de Epidemiologia da Faculdade de Saúde Pública lia USP - Av. Dr. Arnaldo, 715 - São Paulo, SP — Brasil

* Da Disciplina de Figiene Materna do Departamento de Prática Médica em Saúde Pública da Faculdade de Saúde Pública da USP - Av. Dr. Arnaldo, 715 - São Paulo, SP Brasil 
LALRENTI, R. et al. - Mortalidade perınatal em Sāo Paulo, Brasıll. Rev. Saúde públ., S Paulo, 9:115-24, 1975.

natal. A mortalidade perinatal pode assim ser definida como a relação

ólitos fetais tardios + óbitos de menores de 7 dias

nascidos viros

em que os óbitos fetais tardios são repre. sentados pelas perdas fetais de vinte e oito ou mais semanas de gestação.

0 grande interesse que a mortalidade perinatal desperta nos paises desenvolvidos decorre do fato de a mortalidade, nesses países, se concentrar quase que totalmente no período neonatal. Nos Estados Lnidos, por exemplo, dos óbitos de menores de um ano, a metade referia-se a crianças falecidas com menos de dois dias de vida ${ }^{13}$.

Pode-se verificar que. mesmo naqueles países onde a mortalidade infantil é muito baixa, como Islândia e Suécia. a mortalidade perinatal, apesar de muito mais baixa que nos demais países, não acompanha a mortalidade infantil. Isto significa que, mesmo nos países desenvolvidos, existe um número de óbitos perinatais inevitáveis, mesmo nas condiçôes atuais de avanço científico. Esses óbitos referem-se. em geral. a anomalias congênitas muito graves. a recém-nascidos de peso muito baixo e a "acidentes" do parto. como hemorragias, compressões do cordão e outros. Em outras palavras, pode-se dizer que, dificilmente. haverá uma mortalidade perinatal muito baixa. podendo-se fixar um valor de cerca de 20 óbitos por mil nascidos rivos, como situação quase ideal.

Butter e Bonham 2 e Butter e Albermann ${ }^{1}$. analisando resultados do "British Perinatal Mortality Survey". consideram a causa e o momento do óbito estritamente relacionados entre si. Assim. a mortalidade fetal "ante-partum" deve-se. principalmente, à toxemia gravídica e às hemorragias, ambas as entidades preveníveis a nível de pré-natal. Quanto à diminuição da mortalidade fetal "intra-partum", depende da identificação e trata- mento adequado das gestações de alto risco. seja no pré-natal, com uma concentração de consultas de boa qualidade, seja através da vigilância durante o parto. com a utilização de técnicas de monitorização e avaliação seriada do $\mathrm{pH}, \mathrm{pO}$. e pCO. por exemplo.

A mortalidade no primeiro dia de vida. nos paises desenvolvidos. resume-se quase que somente em crianças de peso insuficiente nascidas antes do tempo e a anomalias congênitas letais. A mortalidade do $2 .^{\circ}$ a $60^{\circ}$ dia dere-se à insuficiência ponderal do recém-nascido, às complicaçóes respiratórias dela decorrentes e às infecções.

Gs níveis de saúde em São Paulo estão longe de serem considerados satisfatórios ${ }^{1 i}$. A mortalidade infantil, que vinha diminuindo até 1961. quando atingiu a cerca de 60 óbitos por mil nascidos vivos, está aumentando, desde então, ano a ano ${ }^{6,-}$

Até o momento, ainda não foi estudada a mortalidade perinatal para São Paulo, seja por dificuldades na obtenção de dados fidedignos - a qualidade de certificação da causa do óbito deixa muito a desejar ${ }^{5.5}$ - seja pela impossibilidade de escoimar, do volume total de óbitos. os referentes a gestaçōes de mulheres não residentes em São Paulo e que para aqui vêm em busca de atendimento médicohospitalar. Além disso. até muito recentemente, o Departamento de Estatística do Estado da Secretaria de Economia e Planejamento, não apurara a natimortalidade por causas.

Silveira ${ }^{15}$ apresentou, recentemente. monografia sobre as perdas fetais do distrito de São Paulo e Siqueira ${ }^{16}$ sobre a mortalidade neonatal.

Nesta apresentação foram reunidos os dados referentes às perdas fetais tardias e à mortalidade neonatal precoce. Serão estudados o valor do coeficiente de mortalidade perinatal, as principais causas de 
LAURENTI, R. et al. - Mortalidade perinatal em São Paulo, Brasil. Rev. Saíde públ., S. Paulo, 9:115-24, 1975.

óbito e a mortalidade segundo a idade da mãe. Outros aspectos da mortalidade perinatal, especialmente aqueles relacionados à atenção pré-natal e ao parto serão objeto de ulterior estudo.

Os objetivos propostos foram, para o Distrito de São Paulo:

- medir o valor real do coeficiente de mortalidade perinatal;

- estudar a mortalidade perinatal segundo as causas da morte;

- estudar a mortalidade perinatal segundo a idade da mãe.

\section{MATERIAL E METODOS}

O material utilizado para o estudo da mortalidade perinatal constituiu-se, de um lado, dos atestados de nascidos mortos e, de outro, das crianças nascidas vivas que chegaram a óbitos nos primeiros seis dias de vida.

Para ambos os grupos, no Departamento de Estatística do Estado, foram selecionados os atestados referentes aos eventos citados e ocorridos no período $1 .^{\circ}$ de junho de 1968 a 31 de maio de 1970 e cujas mães residissem no distrito de São Paulo.

Com relação aos nascidos mortos foram encontrados 5.467 atestados que preenchiam essas condições. O material trabalhado se constituiu da totalidade desses casos e sua análise fez parte de estudo relativo às perdas fetais ${ }^{15}$.

Com referência aos óbitos de crianças menores de sete dias, a metodologia utilizada seguiu a da Investigação Interamericana de Mortalidade na Infância ${ }^{6,11}$, dado que seu estudo foi parte daquela pesquisa ${ }^{16}$. Foram encontrados 1.159 casos de óbitos ocorridos no período neonatal precoce que, segundo a metodologia da Investigação, foram exaustivamente pesquisados através de entrevistas do- miciliares e junto aos médicos e hospitais que cuidaram dos falecidos. Visava-se com isso, entre outros aspectos, medir, exatamente, as reais causas de morte dessas crianças. Esses 1.159 casos representavam uma amostra dos óbitos neonatais precoces, de mães residentes no distrito de São Paulo. $O$ número apresentado neste trabalho (4.926 casos) representa a estimativa feita para toda a população, através da multiplicação do número encontrado pelo fator de amostragem.

0 estudo refere-se, como foi dito, ao distrito de São Paulo; seus resultados, no entanto, podem ser estendidos a todo o município, dado que a população do primeiro representa aproximadamente $90 \%$ da verificada para o município de São Paulo.

\section{RESULTADOS E DISCUSSAO}

O número total de óbitos perinatais ocorridos no período 1-6-68 a 31-5-70 foi de 10.393 , sendo 5.467 perdas fetais tardias ou nascidos mortos e 4.926 ocorridos nos primeiros seis dias de vida. É interessante frisar que esses dados sofreram vários tipos de correção: relativa ao tempo em que se verificou o evento, relativa à residência da mãe e relativa aos casos de nascidos vivos registrados como nascidos mortos e vice-versa.

Para o mesmo período, verificaram-se, para mães residentes no distrito de São Paulo, 247.191 nascimentos vivos, número esse também devidamente corrigido de acordo com a própria metodologia de coleta de dados da Investigação Interamericana de Mortalidade na Infância.

Atendendo aos objetivos propostos por este trabalho, são os seguintes os resultados a serem apresentados e discutidos:

\section{1) Coeficiente de mortalidade perinatal}

$O$ coeficiente de mortalidade perinatal foi calculado para todo o período, apre- 
LAURENTI, R. et al, - Mortalidade perinatal em Sāo Paulo, Brasil. Rev. Saúde públ., S. Paulo, 9:115-24, 1975.

sentando-se igual a 42,04 por mil nascidos vivos.

Esse valor é bastante elevado quando comparado ao coeficiente de mortalidade perinatal de países desenvolvidos, onde, conforme se vê através dos dados da Tabela 1 , alguns valores estão ao redor de 20 por mil nascidos vivos.

TA B E L A 1

I.Iortalidade perinatal e infantil (coeficientes por mil nascidos vivos) em alguns paises, em anos próximos a 1967

\begin{tabular}{lcc}
\hline \multicolumn{1}{c}{ Pais } & Perinatal & Infantil \\
& & \\
\hline & & 25,5 \\
Alemanha Oriental & 27,9 & 23,5 \\
Alemanha Ocidental & 20,8 & 13,7 \\
Islândia & $\mathbf{3 5 , 3}$ & 38,4 \\
Hungria & 36,8 & 34,3 \\
Itália & 43,6 & 64,7 \\
Portugal & 18,9 & 12,6 \\
Suécia & & \\
\hline
\end{tabular}

Fonte: Seminário sobre la Prevencion de la Morbilidad y la Mortalidad Perinatales. Tours, 1969 1:

Em relação ao momento do óbito perinatal $52,60 \%$ referem-se aos nascidos mortos e $47,40 \%$ aos óbitos neonatais precoces. Ao desdobrar o coeficiente de mortalidade perinatal em seus componentes. verifica-se que o de natimortalidade foi da ordem de 22,12 por mil nascidos vivos e o de mortalidade neonatal preroce igual a 19,93 por mil nascidos vivos.

\section{2) Mortalidade perinatal segundo causas}

A tabulação das causas de morte foi feita de acordo com a oitava Revisão da Classificação Internacional de Doenças. $1965^{11}$.

Para maior facilidade de estudo as causas constantes da Lista Tahular foram reunidas em grupos e suh-orupos. a saher: a) Causas Perinatais: correspondentes ao capítulo XV da Classificação Internacional de Doenças, abrangendo:

- causas maternas associadas à gravidez (categorias 760 e 76 J.)

- causas maternas próprias da gravidez (categorias 762 e 763 )

- partos distócicos e traumatismos obstétricos (categorias 764, 765, $766,767,768$ e 772 )

- outras complicações da gravidez e do parto (categoria 769)

- afecções da placenta e cordão umbelical (categorias 770 e 771 )

— interrupção da gravidez (categoria 773 )

- doença hemolítica de recém-nascido (categorias 774 e 775 )

- afeçẽos anóxicas e hipóxicas (categoria 776)

- prematuridade (categoria 777)

- outras afecções do feto ou recémnascido (categoria 778)

- morte fetal de causa desconhecida (categoria 779)

b) Anomalias Congênitas: correspondendo ao capítulo XIV da Classificação Internacional de Doenças;

(i) Doenças Infecciosas: correspondendo ao capítulo I da Classificação Internacional de Doenças;

d) Doenças Respiratórias: correspondendo ao capítulo VIII da Classificação Internacional de Doenças;

e) Acidentes, envenenamentos e violências: correspondendo ao capítulo XVII, Chave E, da Classificação Internacional de Doenças.

A Tahela 2 mostra a distribuição dos nascidos mortos e dos óbitos de menores de sete dias, segundo esses grupos e subgrupos. bem como o coeficiente de mortalidade perinatal calculado para essas c'ausas e por cem mil nascidos vivos. 
LAURENTI, R. et al. - Mortalidade perinatal em São Paulo, Brasill. Rev. Saúde públ., S. Paulo, 9:115-24, 1975.

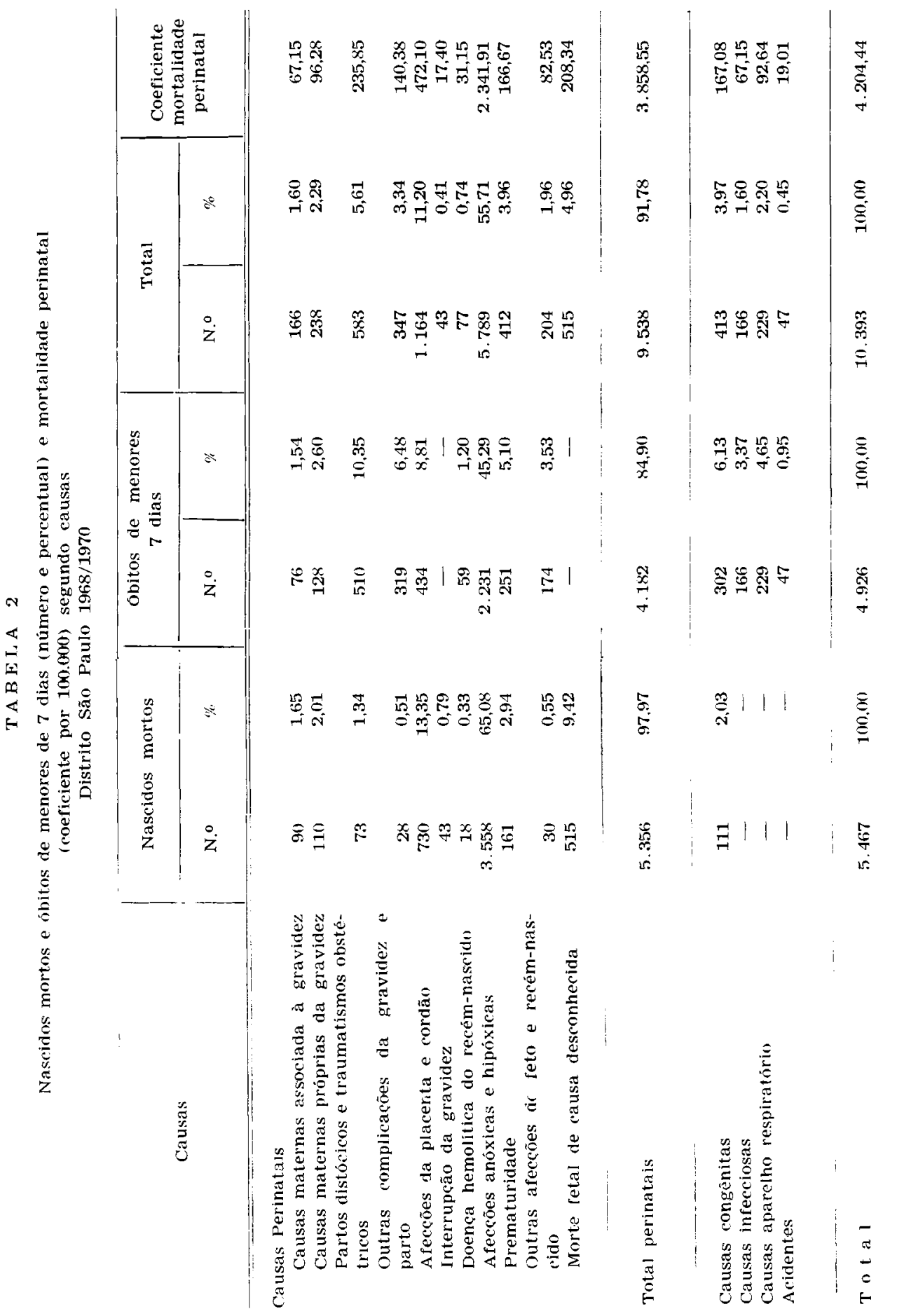


LAURENTI, R, et al. - Mortalidade perinatal em São Paulo, Brasil. Rev. Saúde públ., S. Paulo, 9:115-24, 1975.

Chama a atenção, nessa Tabela, o número excessivo de nascidos mortos por afecções anóxicas e hipóxicas - categoria 776 da Lista Tabular - representado por $65,08 \%$ do total de perdas tardias. Evidentemente, a primeira crítica que poderia ser feita é a relativa à má qualidade da certificação médica da causa do óbito, dado que a anóxia, em si, não representa causa básica de morte, mas sim uma causa final, conseqüente a outra afecção, quer materna, quer fetal. Entretanto, mesmo com todas as informações adicionais obtidas através da minuciosa metodologia $\mathrm{da}$ Investigação Interamericana de Mortalidade na Infância, não se conseguiu, em $45,29 \%$ dos óbitos ocorridos no período neonatal precoce, chegar a outro diagnóstico que não a própria anóxia. Este dado apresenta-se ainda bastante elevado e isso se deve, entre outros fatores, ao baixo percentual de autópsias, tanto entre os nascidos mortos $(29,61 \%)$ quanto em relação aos óbitos de menores de 7 dias, aproximadamente $10 \%$. 0 coeficiente de mortalidade perinatal por afecções anóxicas e hipóxicas foi igual a 2.341,91 por cem mil nascidos vivos.

Evidencia claramente essa Tabela o pequeno número de óbitos perinatais por causas maternas $(3,89 \%$, quando se consideram todas as afecções da mãe, próprias (762-763) ou associadas (760-761) à gravidez). Esse achado é surpreendente, dado que, ao menos teoricamente, deveria se concentrar neste grupo, a maior parte dos óbitos perinatais.

Os coeficientes que medem essa mortalidade foram respectivamente iguais a 67,15 por cem mil nascidos vivos para as causas associadas à gravidez e 96,28 por cem mil nascidos vivos para as toxemias e infecçōes maternas.

Com relação à mertalidade perinatal por distócias do parto e traumatismos obstétricos nota-se grande diferença entre o que ocorreu nos nascidos mortos e nos óbitos de menores de 7 dias, representados por, respectivamente, $1,34 \%$ e
$10,35 \%$ de seus totais. Essa disparidade foi, sem dúvida, decorrente da busca minuciosa das reais causas de óbito que, como parte da Investigação de Mortalidade na Infância, sofreram os óbitos neonatais precoces. Sua metodologia, por não se basear, como foi visto, somente nas causas declaradas nos atestados de óbito, permitiu identificar, para este grupo específico, aproximadamente oito vezes mais de casos ${ }^{12}$. Assim, o coeficiente de mortalidade perinatal por doenças e lesões do parto apresentou-se igual a 235,85 por cem mil nascidos vivos. Cumpre salientar que o componente neonatal precace desse coeficiente $(206,32$ por cem mil nascidos vivos) é alto quando comparado com áreas mais desenvolvidas, como por exemplo, Sherbrooke, no Canadá e Califórnia, nos Estados Unidos, onde seu valor foi, aproximadamente, quatro vezes menor ${ }^{11}$.

As causas agrupadas sob os números 770 e 771 , que constituem as afecções da placenta e cordão, apresentaram um coeficiente de mortalidade perinatal igual a 472,10 por cem mil nascidos vivos. Esse valor, ao que tudo indica, está também subestimado, pois todos os problemas ligados à placenta e ao cordâo levam sempre a uma anóxia, imaginando-se, portanto, que um certo número destes casos tenham sido computados na categoria 776.

Surpreendente também foi o aparecimento da categoria 773 que engloba todos os tipos de aborto. Entende-se por aborto toda interrupção de gravidez até a vigésima sétima semana ${ }^{9}$. Se por outro lado sabe-se que nascido morto é o produto de concepção expelido ou extraído sem vida do corpo da mãe, com gestação de 28 ou mais semanas de duração, evidentemente, a causa citada - interrupção da gravidez - não deveria aparecer para o grupo considerado.

Com relação à prematuridade, o coeficiente encontrado $(166,67$ por cem mil nascidos vivos) não representa a sua freqüência nos óbitos perinatais, mas sim, 
LAURENTI, R. et al. - Mortalidade perinatal em São Paulo, Brasil. Rev. Saúde públ., S. Paulo, 9:115-24, 1975.

refere-se somente aos casos em que a prematuridade foi a única causa declarada nos atestados de nascidos mortos ou encontrada nos óbitos neonatais precoces.

A título de ilustração pode-se salientar que, do total de óbitos neonatais ocorridos em São Paulo, no período da "Investigação", cerca de $60 \%$ referiram-se a crianças nascidas com peso inferior a 2.500 gramas $^{16}$.

A categoria 779 representada pela "morte fetal de causa desconhecida", "macerado" ou simplesmente "nascido morto", sem outra especificação, ocorreu em aproximadamente $10 \%$ dos casos de nascidos mortos, número que levou a um coeficiente de mortalidade perinatal, por essa causa, igual a 208,34 por cem mil nascidos vivos. Evidentemente, também aqui, se melhores diagnósticos fossem feitos e/ou declarados, esta categoria tenderia, praticamente, a desaparecer.

As anomalias congênitas foram responsáveis por $3,97 \%$ do total de causas para o período perinatal. Esse percentual, embora não seja grande, está a merecer acurada atenção de todos quanto se preocupam com o assunto. A rigor, pode-se afirmar que entre os rascidos mortos, o número apresentado está subestimado pois, principalmente dado o pequeno número de autópsias realizadas (sendo que a maioria delas levou apenas ao diagnóstico "nascido morto, macerado"), em grande parte dos casos, somente as anomalias visiveis à simples inspeção (anencefalia, espina bífida, hidrocefalia, por exemplo) eram registradas. De fato, comparando os valores apresentados pelos dois componentes da mortalidade perinatal, verifica-se ser mais alta a proporção do grupo relativo aos óbilos neonatais precoces, bem como mais elevado seu coeficiente, quando, a rigor, deveria ocorrer - contrário. Isso se rerificou dado que muitas anomalias congênitas, principalmente as do aparelho cárdio-circulatório, puderam ser diagnosticadas devido ao simples fato da criança ter nascido viva. Seguramente, se possível uma depuração do diagnóstico, a natimortalidade por malformações congênitas deveria apresentar valor bastante próximo do grupo neonatal precoce, o que, na realidade, não ocorreu (respectivamente 44,90 e 127,17 por cem mil nascidos vivos).

A mortalidade perinatal por doenças infecciosas se deveu somente a casos verificados no período neonatal precoce. 0 coeficiente de mortalidade perinatal por essa causa apresentou-se igual a 67,15 por cem mil nascidos vivos, número que pode ser considerado bastante elevado, justificando, portanto, uma maior atenção ao problema. Pôde ser verificado ${ }^{16}$ que entre os menores de 7 dias $51,28 \%$ dos óbitos deveram-se à diarréia, $25,64 \%$ à sífilis congênita, $20,51 \%$ à septicemia e $2,56 \%$ à monilíase. Chama particularmente a atenção o aparecimento da enfermidade diarréica nesse grupo etário. A Investigação Interamericana de Mortalidade na Infância, que permitiu comparar dados de quinze áreas das Américas, mostrou que apenas a Província de San Juan na Argentina apresentou coeficiente por diarréia mais elevado que São Paulo; é importante frisar ainda que em Sherbrooke e na Califórnia não se verificou nenhum caso de óbito por essa causa, nesse grupo etário ${ }^{11}$.

Relativamente à sífilis congênita, o percentual de ocorrência é também bastante elevado, dado que essa causa constitui entidade perfeitamente evitável. Este é, assim, mais um dado que evidencia a precariedade da atenção pré-natal.

Com relação às doenças do aparelho respiratório, a mortalidade perinatal foi também representada somente pelos óbitos de crianças menores de 7 dias. 0 
LAURENTI, R. et al. - Mortalidade perinatal em São Paulo, 3rasil. Revi. Saüde pübl., S. Paulo, 9:115-24, 1975.

coeficiente se mostrou igual a 92,64 por mil nascidos vivos e a causa predominante, nesse grupo, foi a broncopneumonia.

Os acidentes, envenenamentos e violências apareceram em pequeno número, levando a um coeficiente de 19,01 por cem mil nascidos vivos. Todos os casos verificados ocorreram no período neonatal precoce, sendo, a aspiração e ingestão de alimentos a causa responsável pela maioria deles. Quanto aos acidentes como causa de natimortalidade, ocorreram alguns casos, porém, por disposição da Classificação Internacional de Doenças, devem eles ser classificados como "traumatismos da mãe, afetando o produto da concepção" e computados na categoria 761.

\section{3) Mortalidade perinatal segundo a idade da mãe}

Vários trabalhos têm demonstrado existir uma relação entre a mortalidade neonatal e a idade da mãe, evidenciando que o aumentar da idade faz aumentar essa mortalidade $2,3,11,1$ t. Relativamente aos nascidos mortos os coeficientes seguiram a tendência mostrada nos referidos trabalhos: a possibilidade ou ocorrência de perdas fetais tardias foi maior nas mulheres de menos de 20 anos do que naquelas dos grupos etários seguintes ( 20 a 24 e 25 a 29 anos), aumentando depois com o crescer da idade e atingindo seu valor máximo no grupo de mulheres entre 40 e 44 anos $^{15}$.

Com referência à mortalidade perinatal. nas mulheres de menos de 20 anos. o coeficiente foi igual a 43,56 por mil nascidos vivos, valor próximo ao coeficiente de mortalidade perinatal medido para o conjunto de todas as idades.

Feldstein ${ }^{4}$ mostra que, nesse grupo, a mortalidade perinatal mais elevada se deve a outros fatores que não a idade; cita especificamente as condições sócio-econômico-culturais, dado que as mulheres de estado social mais haixo costumam. em geral, dar à luz mais cedo.

No grupo etário de 20 a 29 anos. observou-se o mais baixo valor do coeficiente (31,90 por mil nascidos vivos), seguindo, portanto, a já referida tendência de representar as idade de menor risco.

Para as mulheres de 30 a 39 anos, o coeficiente aumentou para 46,84 por mil nascidos vivos, continuando a crescer para o grupo etário seguinte ( 40 a 49 anos). onde atingiu o valor de 63,33 por mil nascidos vivos.

Dessa forma, as mulheres com idades compreendidas entre 40 e 49 anos constituem grupo de alto risco e estão, portanto, a merecer maior atenção dos serviços de atendimento pré-natal e ao parto.

A Figura 1 mostra a curva da mortalidade perinatal segundo a idade da mãe.

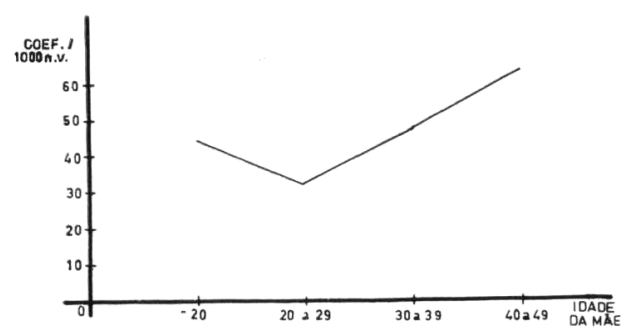

Fig, 1 - Mortalidade perinatal segundo a idade da mãe (coeficiente por mil nascidos vivos).

\section{CONCLUSOES}

1..$^{\circ}$ A mortalidade perinatal para o distrito de São Paulo (coeficiente corrigido) no período 1-6-68 a 31-5-70 foi igual a 42,04 por mil nascidos vivos, valor esse bastante elevado quando comparado a áreas desenvolvidas;

$2 .^{\circ}$ o coeficiente de mortalidade perinatal por algumas causas poderia ser bastante reduzido com a simples redução da mortalidade por al- 
LALRENTI, R. et al. - Mortalidade perinatal em São Paulo, Erasil. Rer. Saúde públ,, S Paulo, 9:115-24, 1975.

gumas causas específicas evitáveis. seja a nível de pré-natal (sífilis congênita, doenças próprias ou associadas à gravidez), do parto (distócias, traumatismos obstétricos e anóxias) ou da atenção ao recém-nascido (causas infecciosas, do apare- lho respiratório, hemorragias e mesmo muitas anóxias);

3.) o coeficiente de mortalidade perinatal segundo a idade da mãe mostrou que o risco varia com a idade. apresentando-se maior nas mulheres de 40 a 49 anos.

RSPU-B/253

Laurenti, R. et al. - [Perinatal mortality in S. Paulo, Brazil]. Rev. Saúde públ., S. Paulo, 9:115-24, 1975.

Summary: Perinatal mortality in S. Paulo, over a yeriod of two years, was analysed. The study took in all death certificates of the stillborn and a sample of children under a week of age. For the latter the methodology used was by interviewing phisicians and hospitals that cared for the deceased. The perinatal mortality rate was 42,05 per thousand live births. This value is really high when compared with those of developed areas. Nevertheless it can be reduced once the specific rates for some of the avoidable diseases be reduced by proper pre-natal care (congenital syphilis, illness pertaining to or associated with pregnancy). This can also be done by improving care at delivery (Distocias, obstetrical traumatism and anoxia) and towards the newlyborn (infeccious diseases, respiratory diseases, haemorrages and anoxia). The perinatal mortality rate varies with the age of the mother, the risk being largest in women between 40 and 49 years of age.

Uniterms: Perinatal Mortality, S. Paulo, Brazil. Vital statistics. Neonatal mortality. Natimortality.

\section{REFERENCIAS BIBLIOGRAFICAS}

1. BUTLER, N.R. \& ALBERMAN, E.D. Perinatal problems. Edimburg, Livingstone, 1969.

2. BUTLER, N.R. \& BONHAM, D.G. Perinatal mortality. Edimburg, Livingstone, 1963.

3. CARVAlHeiro, C.D.G. - Estudo da mortaliaade perinatal. Ribeirão Preto, 1970. [Tese de doutoramento].

4. FELDSTEIN, M.S. \& BUTLER, N.R. Analysis of factors affecting perinatal mortality: a multivariate statistical approach. Brit. J. prev. soc. Med., 19:128-34, 1965.

5. FONSECA, L.A.M. \& LAURENTI, R. - A qualidade da certificação médica da causa de morte em São Paulo, Brasil. Rev. Saúde públ., S. Paulo, 8:21-9, 1974.

6. LAURENTI, R. - A "Investigação Interamericana de Mortalidade na In- fância" em São Paulo, Brasil. Rev. Saúde publ., S. Paulo, 3:225-9, 1969.

7. LAURENTI, R. \& SIQUEIRA, A.A.F. de -0 problema da mortalidade neonatal em São Paulo, Brasil. Rev. Saúde públ., S. Paulo, 6:45-55, 1972.

8. Milanesi, M.L. \& LAURENTI, R. O Estudo Interamericano de Mortalidade em São Paulo. Estudo atual da certificação médica da causa de óbito no distrito da Capital. Rev. Ass. méd. Bras., 10:111-6, 1964.

9. NACIONES UNIDAS - Manual de metodos de estadisticas vitales. New York, 1955. (Estudos Metodológicos. Série Is, n.o 7).

10. ORGANIZAÇÃO PANAMERICANA DA SAŨDE - Manual da Classificação Estatística Internacional de Doen. ças, Lesões e causas de óbito. $8 .^{\mathrm{a}}$ Revisão. Washington, D.C., 1969. (Publ. cient. 190) 
LALRENTI, R. et al. - Mortalidade perinatal em Sāo Paulo, Brasil. Rev. Saúde puibl., S. Paulo, 9:115-24, 1975.

11. PUFFER, R.R. \& SERRANO, C, - Caracteristicas de la mortalidad en la niñes. Washington, D.C., Organizacion Panamericana de la Salud, 1973. (Publ, cient., 262).

12. SEMINARIO SOBRE IA PREVENCION DE LA MORBILIDAD Y LA MORTALIDAD PERINATALES, Tours, 1969. Informe. Ginebra, Organizacion Mundial de la Salud, 1972. (Cuadernos de salud publica, 42).

13. SHAPIRO, S. et al. - Infant and perinatal mortality in the United States. Washington, D.C., Public Health Service, 1965. (Vital and Health Statistics. Serie $\left.3, n^{\circ} 4\right)$.

14. SHAPIRO, S. et al. - Infant, perinatal maternal and childhood mortality in the United States. Cambridge, Mass., 1968.

15. SILVEIRA. M.H. - Perdas fetais do distrito de São Paulo. São Paulo, 1974. [Monografia de Mestrado Faculdade de Saúde Pública - USP].

16. SIQUEIRA, A.A.F. - Mortalidade neonatal e prematuridade. São Paulo, 1974. [Monografia de Mestrado Faculdade de Saúde Pública — USPJ.

17. YUNES, J. \& BROMBERG, R. - Os niveis cle saúde na região da Grande são Paulo. Rev. Saúde públ., S. Paulo, 4:167-88, 1970.

Recebido para publicacio en 14-09-1975 Aprovado para publicasao em 0/4-0.4-1975 